Article

\title{
Optimization and Characterization of High-Harmonic Generation for Probing Solid Density Plasmas
}

\author{
Jayanath C. P. Koliyadu ${ }^{1,2}$, Swen Künzel ${ }^{1, *}$, Thomas Wodzinski ${ }^{1}$, Barbara Keitel ${ }^{3}$, \\ Joana Duarte ${ }^{1}$, Gareth O. Williams ${ }^{1}$, Celso P. João ${ }^{1}$, Hugo Pires ${ }^{1}$, Victor Hariton ${ }^{1}$, \\ Mario Galletti ${ }^{1}$, Nuno Gomes ${ }^{1}$, Gonçalo Figueira ${ }^{1}$, João Mendanha Dias ${ }^{1}$, \\ Nelson Lopes ${ }^{1}$, Philippe Zeitoun ${ }^{2}$, Elke Plönjes ${ }^{3}$ and Marta Fajardo ${ }^{1}$
}

1 GoLP/Instituto de Plasmas e Fusão Nuclear, Instituto Superior Técnico, 1049-001 Lisboa, Portugal; jayanathkoliyadu@tecnico.ulisboa.pt (J.C.P.K.); thomas.wodzinski@tecnico.ulisboa.pt (T.W.); joana.r.duarte@tecnico.ulisboa.pt (J.D.); gareth.williams@tecnico.ulisboa.pt (G.O.W.); celsopaivajoao@ist.utl.pt (C.P.J.); hugo.pires@ist.utl.pt (H.P.); victor.hariton@ist.utl.pt (V.H.); mario.galletti@tecnico.ulisboa.pt (M.G.); ngomes@ipfn.ist.utl.pt (N.G.); goncalo.figueira@ist.utl.pt (G.F.); joao.m.dias@tecnico.ulisboa.pt (J.M.D.); Nelson.Lopes@ist.utl.pt (N.L.); marta.fajardo@ist.utl.pt (M.F.)

2 Laboratoire d'Optique Appliquée, ENSTA-Paristech, CNRS, Ecole Polytechnique, UMR 7639, 828 Boulevard des Maréchaux, 91128 Palaiseau, France; philippe.zeitoun@ensta-paristech.fr

3 Deutsches Elektronen-Synchrotron DESY, Notkestrasse 85, 22607 Hamburg, Germany; barbara.keitel@desy.de (B.K.); elke.ploenjes@desy.de (E.P.)

* Correspondence: swen.kunzel@tecnico.ulisboa.pt

Received: 31 January 2017; Accepted: 23 March 2017; Published: 30 March 2017

\begin{abstract}
The creation of high energy density plasma states produced during laser-solid interaction on a sub-picosecond timescale opens a way to create astrophysical plasmas in the lab to investigate their properties, such as the frequency-dependent refractive index. Available probes to measure absorption and phase-changes given by the complex refractive index of the plasma state are extreme-UV (EUV) and soft X-ray (XUV) ultra-short pulses from high harmonic generation (HHG). For demanding imaging applications such as single-shot measurements of solid density plasmas, the HHG probe has to be optimized in photon number and characterized in intensity and wavefront stability from shot-to-shot. In an experiment, a coherent EUV source based on HHG driven by a compact diode-pumped laser is optimized in photons per pulse for argon and xenon, and the shot-to-shot intensity stability and wavefront changes are characterized. The experimental results are compared to an analytical model estimating the HHG yield, showing good agreement. The obtained values are compared to available data for solid density plasmas to confirm the feasibility of HHG as a probe.
\end{abstract}

Keywords: XUV; high-harmonic generation; high energy density physics; diode-pumped lasers; pump-probe

\section{Introduction}

High energy density plasmas are created in high power laser facilities worldwide. When a high power laser (Intensity $>10^{14} \mathrm{~W} / \mathrm{cm}^{2}$ ) interacts with a solid target, the target undergoes a transition from solid to plasma state. With ultra-short laser heating, the plasma passes through an intermediate state called warm dense matter (WDM), in which the density is nearly equal to solid density, but the temperature is on the order of $\sim 10^{5} \mathrm{~K}$. This transient state exists for picoseconds [1,2]. Knowing how this transition is created, and understanding the dynamics of this intermediate high energy density plasma state is important for modeling how the laser energy is absorbed by solids. In return, this helps in understanding the laser energy deposition on targets in inertial confinement fusion (ICF) [3] and for 
modeling the equations of state of astrophysical objects where the main constituent is WDM $[1,2,4]$. For high-density plasma states, it is important to have a probe which can penetrate the plasma with a sub-picosecond temporal resolution. Available probes are $\mathrm{X}$-rays produced by free-electron lasers (FEL) [5], extreme-UV (EUV) and soft X-ray (XUV) [6] pulses produced by the process of high-harmonic generation (HHG), and soft X-ray lasers seeded by HHG [7,8]. Although X-rays from FELs can provide coherent XUV pulses with a high photon number $\left(2 \times 10^{13}\right.$ photons/shot $)$ [9] and coherence and peak intensities up to $10^{18} \mathrm{~W} / \mathrm{cm}^{2}$ [10], its major drawbacks are size, cost, and availability. The widely-available source of coherent XUV pulses is HHG, which also has a high spatial coherence and additionally supports pulse durations up to $\sim 100$ attoseconds [11]. Compared to FELs, the photon number of HHG is smaller $\left(10^{9}\right.$ photons/shot), but it is sufficient to carry out single-shot experiments [12]. In order to use XUV pulses from HHG for different applications, it is important to optimize the photon number and to characterize the stability and spatial properties. For experiments like plasma probing [7,13], holography [14], coherent diffraction imaging [12], and X-ray laser seeding [15], it is necessary to have spatially characterized and energy-optimized XUV pulses. Optimization in photon number and wavefront is achieved by adjusting the phase matching conditions of the different high-harmonic orders [16-19]. To take full advantage of the ultrafast resolution achievable with HHG, synchronization between the high-harmonics and pump pulse from the high power laser beam-line is crucial. Therefore, HHG and pump pulse ideally originate from the same laser. A significant fraction of current high power lasers are flash-lamp-pumped, which results in a low pumping efficiency. Additionally, the active medium is heated up unnecessarily and only low repetition rates can be supported. An emerging key technology is diode-pumped laser systems, which overcome both limitations thanks to their unparalleled electrical-to-optical efficiency, and allow more compact setups that require less maintenance.

In the presented work, we use a diode-pumped $\mathrm{Yb}: \mathrm{CaF}_{2} / \mathrm{Yb}$ :YAG laser for HHG. The advantage of this laser system is the possibility of achieving high pulse energies at a high repetition rate [20,21], which allows for the simultaneous creation of a plasma and HHG. However, the laser pulses from $\mathrm{Yb}$ amplifiers are relatively long compared to conventional laser systems based on titanium-sapphire amplifiers, for which HHG is typically optimized in photon number and wavefront [22]. The number of photons and stability of HHG are optimized for the $\mathrm{Yb}$ amplifier-based laser system delivering $\sim 1$ ps pulses, and the wavefront is characterized. The experimental results for the photon yield in HHG are compared to an analytical model. Finally, the prospects for probing solid density plasmas with the obtained high-harmonic pulses are discussed.

\section{Experimental Setup}

Figure 1 shows a block diagram of the HHG experimental setup in the Laboratory for Intense Lasers (L2I) at the Instituto de Plasmas e Fusão Nuclear (IPFN). The in-house built chirped pulse amplification system consists of two diode-pumped amplification stages [23]. A regenerative amplifier with a $\mathrm{Yb}: \mathrm{CaF}_{2}$ crystal and a multi-pass amplifier with a Yb:YAG crystal are used as amplification stages, and both stages are pumped with diodes. The output of the laser system after compression is $\sim 30 \mathrm{~mJ}$ pulse energy with a pulse duration of $\sim 1000 \mathrm{fs}$ at a wavelength of $1030 \mathrm{~nm}$ with a repetition rate of $2 \mathrm{~Hz}$. This technology based on diode-pumped doped $\mathrm{Yb}$ amplifiers has the potential to be upgraded to joule-level pulse energies at a higher repetition rate [21]. This will enable the generation of pump and probe from the same laser. For HHG, a motorised plano-convex lens (f-number $=f / 12$ ) inside the vacuum chamber is used to focus the incoming IR beam onto the gas cell. The generated XUV pulse is filtered from the residual IR pulse using a $150 \mathrm{~nm}$-thick aluminum filter with supporting mesh. After the aluminum filter, an XUV spectrometer is placed which consists of a grazing incidence silica spherical mirror (grazing angle $=5^{\circ}$, radius of curvature $=10 \mathrm{~m}$ ) followed by a gold transmission grating (1000 lines/mm) and an XUV-sensitive CCD camera (Princeton Instruments Pixis XO 1024B, Acton, MA, USA). For direct measurement of the XUV intensity profile, the XUV camera is placed directly after the filters without spectrometer. For wavefront measurement, the XUV camera is replaced 
by a XUV wavefront sensor with alignment for tip/tilt and the vertical/horizontal axis $[24,25]$ and a $300 \mathrm{~nm}$-thick $\mathrm{Al}$ filter without supporting mesh is used.

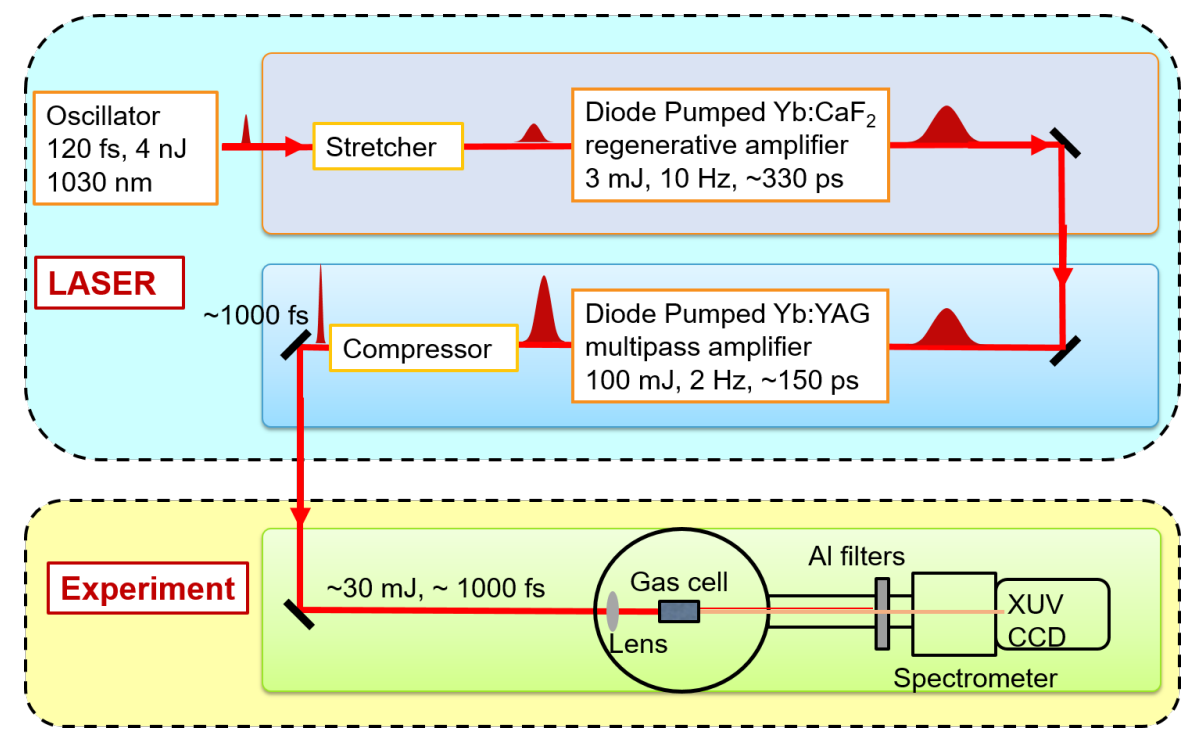

Figure 1. Schematic of high-harmonic generation (HHG) beamline with diode-pumped laser system at the Laboratory for Intense Lasers (L2I), Instituto de Plasmas e Fusão Nuclear (IPFN).

\section{Results}

\subsection{HHG and Optimization}

The high-harmonic photon count was optimized for two noble gases: argon and xenon. The optimization was done by changing the focal length of the lens, gas cell length, position of focus, gas pressure, and beam diameter before lens. The optimized total energy of all harmonic orders was calculated by considering the quantum efficiency and gain of the camera and central frequency of the high-harmonic spectrum. Furthermore, the Al filter used with a thickness of $150 \mathrm{~nm}$ on a nickel support grid was cross-calibrated with a second $\mathrm{Al}$ filter, which resulted in a transmission of $40 \%$ for wavelengths $\sim 10-60 \mathrm{~nm}$. In the case of both gases, the aperture before the lens was reduced to $9 \mathrm{~mm}$ and a pulse energy of $\sim 10 \mathrm{~mJ}$ measured after the aperture. The gas-cell had a length of $10 \mathrm{~mm}$, and the focus was placed at the exit of the gas-cell for optimized HHG. The optimization parameters and obtained results are listed in Table 1.

Table 1. Optimized operating conditions for HHG in argon and xenon calculated for the whole $\mathrm{HH}$ spectrum.

\begin{tabular}{cccccc}
\hline Gas & Rayleigh Length & Focal Length & Pressure & HH Photon Number & HH Energy \\
\hline Units & $\mathrm{mm}$ & $\mathrm{mm}$ & mbar & Photons per Shot & $\mathrm{nJ}$ \\
\hline Argon & 3.3 & 500 & 55 & $7 \times 10^{7}$ & $0.44 \pm 0.05$ \\
Xenon & 6.4 & 700 & 15 & $3.6 \times 10^{8}$ & $1.44 \pm 0.16$ \\
\hline
\end{tabular}

Figure 2 shows the optimized high harmonic beam cross-section and spectrum for argon and xenon. The periodic structure in the high harmonic beam cross-section results from a near-field diffraction effect from the nickel support grid of the filter.

The spatial profile and shot-to-shot stability of the high harmonics beam from argon and xenon was also measured, and the obtained values are listed in Table 2. Figure 3 shows the shot-to-shot stability analysis. 


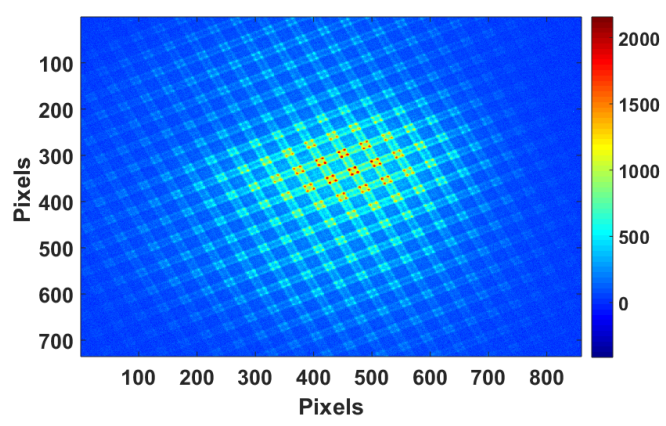

(a)

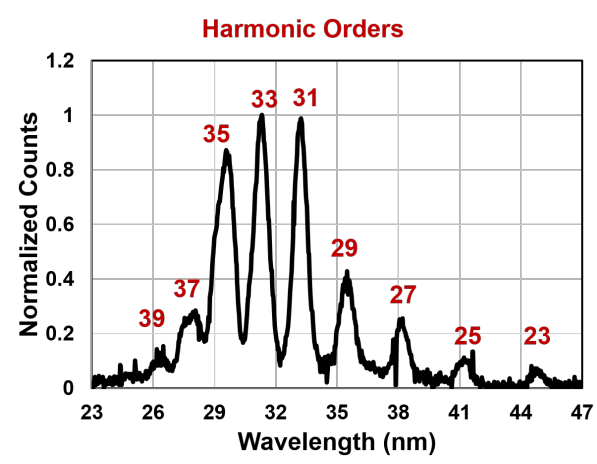

(c)

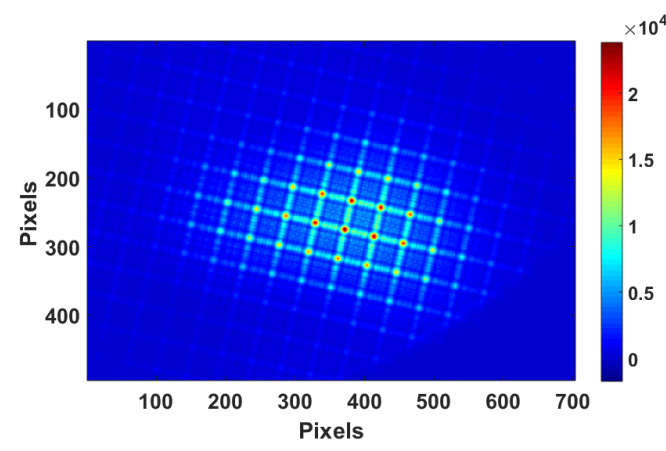

(b)

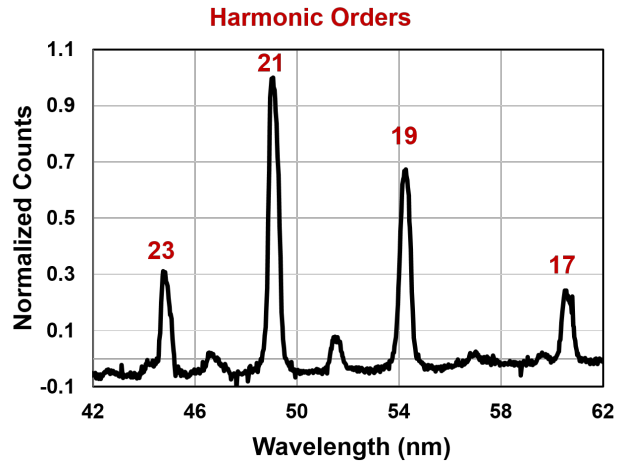

(d)

Figure 2. Beam cross-section $(\mathbf{a}, \mathbf{b})$ and spectrum $(\mathbf{c}, \mathbf{d})$ for HHG from argon and xenon respectively under optimized conditions in terms of number of photons and divergence. The smaller peaks $<0.1$ counts in the xenon spectrum result from the support grid of the grating.

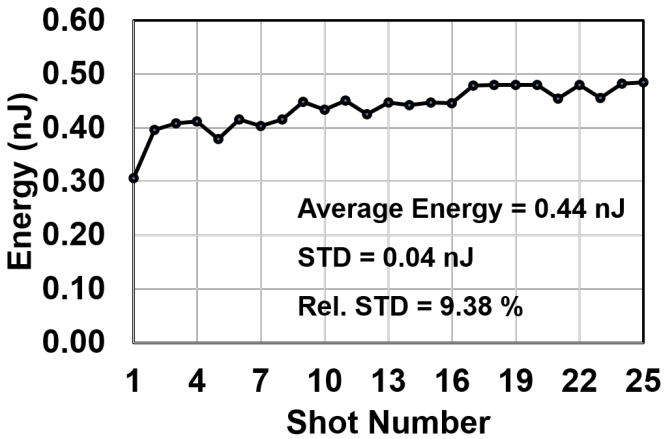

(a)

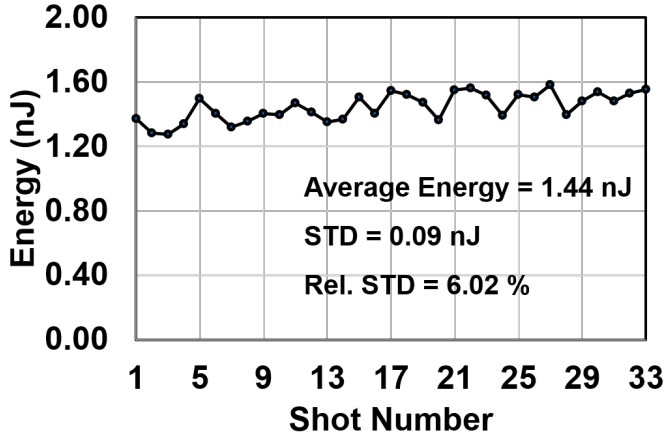

(b)

Figure 3. Single-shot energy stability analysis for high-harmonics generated in (a) argon and (b) xenon.

Table 2. Divergence and shot to shot energy stability of HHG in argon and xenon at L2I (calculated for the whole $\mathrm{HH}$ spectrum).

\begin{tabular}{cccc}
\hline Gas & Divergence Horizontal & Divergence Vertical & Energy Stability \\
\hline Units & mrad & mrad & $\%$ \\
\hline Argon & 2.48 & 2.18 & 9.4 \\
Xenon & 1.89 & 1.25 & 6.0 \\
\hline
\end{tabular}

Experimental results are compared with a numerical calculation of the $\mathrm{HH}$ intensity considering macroscopic phase matching [17]. In non-optimized conditions, HHs were produced in argon using a lens with a focal length of $0.7 \mathrm{~m}$. For the calculation of the $\mathrm{HH}$ intensities, the neutral dispersion, 
plasma dispersion, Gouy phase, atomic phase, and re-absorption were considered. The ionization fraction for the dispersion was calculated using the ADK model [26]. For the experiment, a beam-waist of $64 \mu \mathrm{m}$ was estimated. The focus was $1.3 \mathrm{~mm}$ after the gas-cell, which led to a peak intensity of $\sim 1.5 \times 10^{14} \mathrm{~W} / \mathrm{cm}^{2}$ in the focus and a calculated average ionization fraction of $4.8 \%$ in the gas-cell.

In Figure 4, the experimental measured intensities of $\mathrm{HH}$ order 23 to 39 are integrated and compared to the analytical calculated intensities for different pressures of argon in the gas-cell. Both show a maximum at the same pressure of 55 mbar. Furthermore, the calculated intensities reproduce the experimental curve. However, at pressures above 70 mbar, the calculations show a higher $\mathrm{HH}$ signal than the experimental values. This can be explained by the higher re-absorption of the HHs, since the vacuum system was not able to extract the argon efficiently from the chamber of the gas-cell at these pressures.

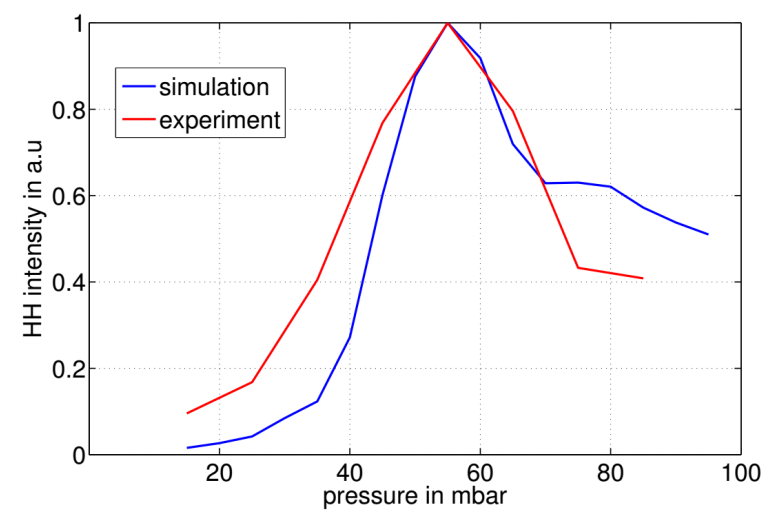

Figure 4. Comparison between simulated and experimental $\mathrm{HH}$ intensity depending on the gas-cell pressure with all $\mathrm{HH}$ orders integrated. The laser is focused $1.3 \mathrm{~mm}$ after the exit surface of the gas-cell.

The ionization fraction for three different laser intensities with varying pulse durations is plotted in Figure 5. It shows that the ionization fraction increases as the pulse duration increases. This results in a higher density of free electrons, which causes the phase-mismatch. Thus, the efficiency of HHG decreases. For the phase matching calculations, the electron density is obtained from calculations using the ADK model, which uses an average ionization. This assumption leads to a good agreement between calculated and experimental values, as shown in Figure 4. This also shows the validity of the model for use in the optimization of the HHG photon yield in the case of long pulse lasers.

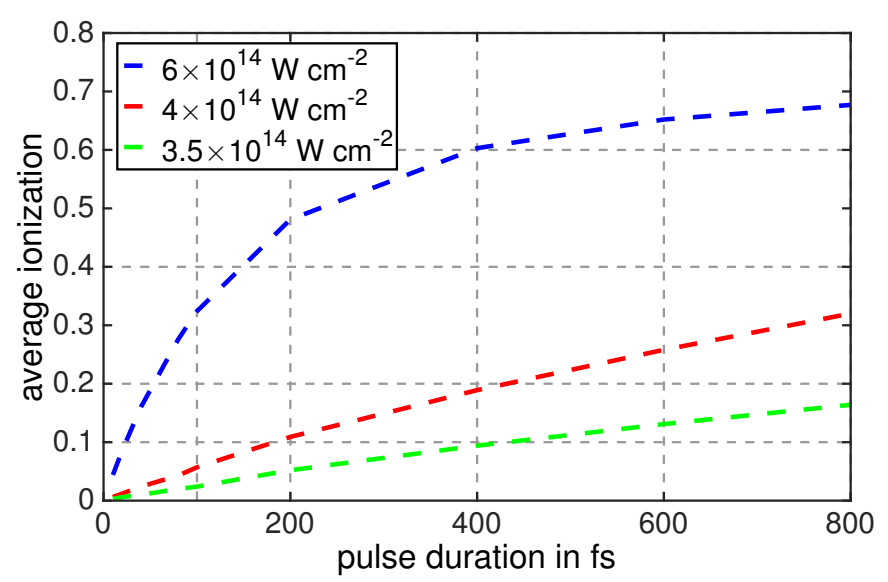

Figure 5. Calculated ionization fractions in argon produced by a laser at three different peak intensities and for different pulse durations. 


\subsection{Wavefront Measurement}

Wavefront (WF) measurements were carried out for HHG in argon with the same optimized generation conditions as listed in Table 1, except the pressure was increased to 65 mbar. This was done to reduce the ellipticity of the beam while still maintaining the photon counts. The Al filter with supporting mesh was replaced by a $300 \mu \mathrm{m}$ filter without mesh with a theoretical transmission of $49 \%$ [27] to avoid diffraction, which disturbs the wavefront measurement. The wavefront data was acquired by integrating the $\mathrm{HH}$ signal for $20 \mathrm{~s}$. In a previous study, it was shown that the integration of wavefronts of multiple shots will not change the root mean square (RMS) wavefront error [19]. The XUV wavefront sensor (WFS) has a Hartmann plate with $57 \times 57$ apertures with a pitch of $250 \mu \mathrm{m}$. The sensitivity of the WFS is $\lambda / 30$ for the spectral range used in the experiment. The full width half maximum (FWHM) of the HH beam diameter is about $2 \mathrm{~mm} \times 3 \mathrm{~mm}$ at the CCD of the WFS. In total, 13 consecutive HH wavefronts were recorded in the same conditions for HHG and compared to estimate the stability of the wavefront. The $\mathrm{HH}$ wavefronts were reconstructed using the software MrBeam [24]. Prior to wavefront reconstruction, a dark image was subtracted and hot pixels were removed for each measurement. Hot pixels arose due to the long exposure time. For the reconstruction, a rectangle of $32 \times 23$ apertures was selected and centered on recorded HH intensity, which covers the full aperture of the beam. An example for a recorded wavefront is shown in Figure 6 . For each measurement, the RMS wavefront error, astigmatism, and coma was calculated; furthermore, the RMS of the beam slopes in the selected area was also calculated. The smallest variation of the RMS wavefront error was $1.14 \mathrm{~nm}$, which corresponds to $\lambda / 28$ and is therefore within the detection limit of the WFS. Additionally, the mean values and their standard deviation were calculated. Figure 7 shows the evolution of the RMS wavefront error, astigmatism, and coma for the different acquisitions, and the mean values are summarized in Table 3.

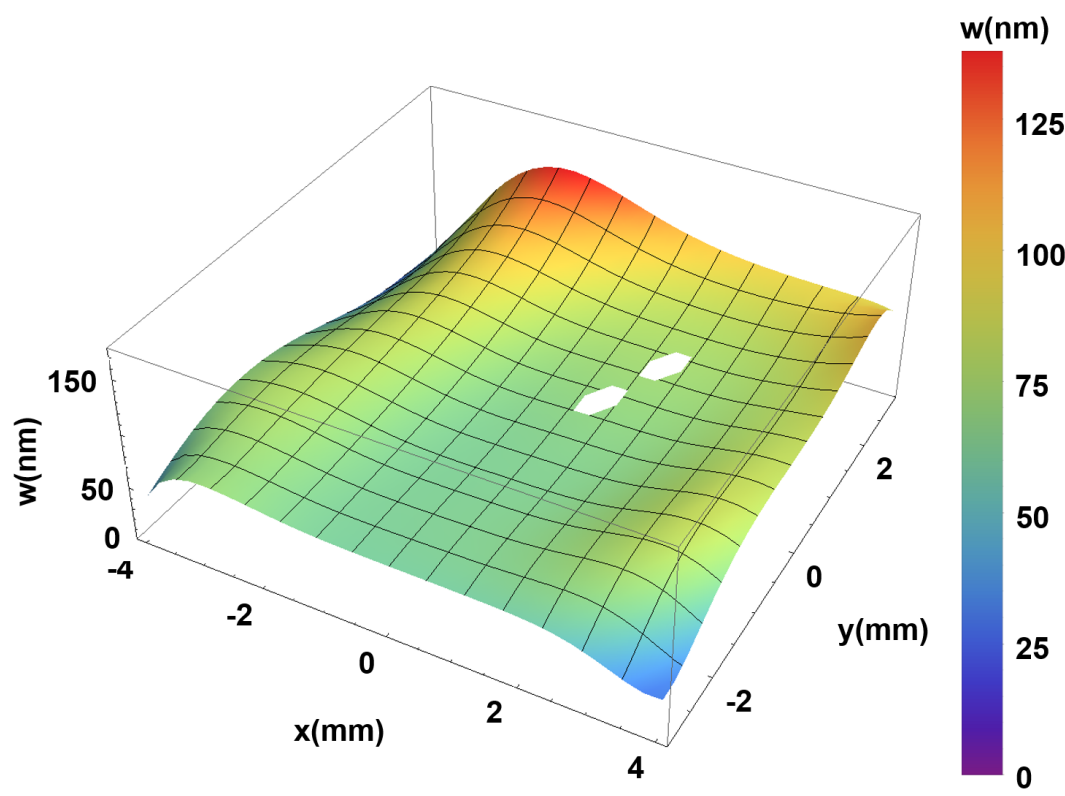

Figure 6. An example of a reconstructed high harmonic wavefront (acquisition 1) measured with the Hartmann type XUV wavefront sensor. One white spot arose from a closed hole in the center of the Hartmann plate. It is used for alignment of the WFS to the beam. The other white spot occurred due to a small particle covering one hole of the plate accidentally. 


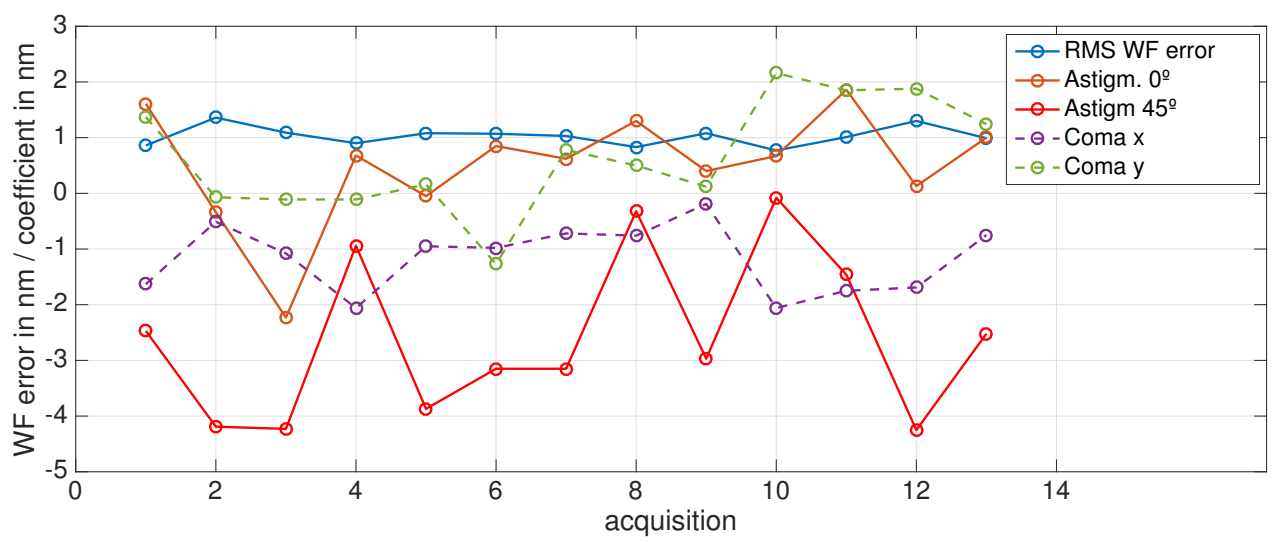

Figure 7. The evolution of the root mean square (RMS) wavefront (WF) error and the Zernike coefficients of the main aberrations.

Table 3. Mean values for the RMS wavefront error and the Zernike coefficients over 13 acquisitions.

\begin{tabular}{ccc}
\hline Parameter & Mean & Standard Deviation \\
\hline RMS Wavefront Error & $4.90 \mathrm{~nm}$ & $1.14 \mathrm{~nm}$ \\
Astigmatism $0^{\circ}$ & $-41.50 \mathrm{~nm}$ & $18.48 \mathrm{~nm}$ \\
Astigmatism $45^{\circ}$ & $5.12 \mathrm{~nm}$ & $9.55 \mathrm{~nm}$ \\
Coma X & $9.37 \mathrm{~nm}$ & $6.86 \mathrm{~nm}$ \\
Coma Y & $-21.85 \mathrm{~nm}$ & $35.03 \mathrm{~nm}$ \\
RMS Slope & $0.60 \mathrm{mrad}$ & $0.01 \mathrm{mrad}$ \\
\hline
\end{tabular}

\section{Discussion}

HHG from argon and xenon were optimized in terms of energy with single shot energies of $0.44 \pm 0.05 \mathrm{~nJ}$ and $1.44 \pm 0.16 \mathrm{~nJ}$, respectively. The XUV pulses from HHG were characterized in spectrum, spatial profile, wavefront, and the shot-to-shot stability in energy and integrated wavefront stability. A shot-to-shot stability of $9.4 \%$ was measured for argon, and $6.0 \%$ for HHG in xenon. From the spectral analysis of the HHG from xenon, we can find that the brightest HH for xenon is order 21 with $\sim 44 \%$ of the total HH energy. In the spectrum of argon $\mathrm{HH}$ order 35, 33, and 31 have nearly equal intensities. All three together share $~ 71 \%$ of the total $\mathrm{HH}$ energy. Analytical calculations for the $\mathrm{HH}$ emission confirm the optimized parameters for pulse energy, gas pressure, focal length, and beam diameter. Therefore, the numerical calculations considering the macroscopic phase matching conditions are an important tool for low repetition rate laser facilities, where extensive optimization scans are not practical.

In [13] values for the absorption and deflection of a XUV probe pulse in Al heated to warm-dense conditions are estimated. The referenced values result from heating $\mathrm{Al}$ with an FEL, however WDM can also be produced with optical lasers as shown in [28-30]. At a temperature of $10 \mathrm{eV}$, an absorption coefficient of $\alpha \approx 4.5 \times 10^{6}$ is estimated for photons with an energy of $30 \mathrm{eV}(\sim 41 \mathrm{~nm})$, which leads to an absorption of $\sim 75 \%$ in a $300 \mathrm{~nm}$-thick foil. More absorption is expected for lower photon energies. In xenon, the $\mathrm{HH}$ order 23 with a wavelength of $44.7 \mathrm{~nm}$ has an energy of $13 \%$ of the whole spectrum, which corresponds to $\sim 0.18 \mathrm{~nJ}$. This corresponds to $\sim 2 \times 10^{6}$ counts in the XUV CCD camera used in the experiment. For an area of $100 \times 100$ pixels on the CCD, this relates to 200 counts per pixel, which is sufficient to be recorded in a single-shot acquisition. The deflection $\phi(x)$ of a beam in a plasma is given by the gradient of the refractive index $\nabla n$ and the length of the plasma $L$ by

$$
\phi(x)=\int_{0}^{L} \nabla n(x, z) d z,
$$


where $x$ is the transverse direction to the plasma and $z$ is the perpendicular direction. Following [13], the largest deflection of an $\mathrm{HH}$ probe beam-larger than the plasma created at WDM conditions-is between the cold $\mathrm{Al}$ foil and warmest part of the solid plasma at a temperature of $10 \mathrm{eV}$. For a plasma with a diameter of $20 \mu \mathrm{m}$, a maximum beam deflection of $0.93 \mathrm{mrad}$ is expected under these conditions. The measured mean slope of the characterized HH source was $0.6 \mathrm{mrad}$ with a standard deviation of $10 \mu \mathrm{rad}$, which is smaller than the expected beam deflections. Therefore, the plasma-induced deflections are more significant than the fluctuations of the probe beam.

The narrower amplification bandwidth of diode-pumped $\mathrm{Yb}$ amplifiers leads to a Fouriertransform-limited pulse duration of a few hundred fs [31,32], which is not a typical parameter space for the optimization of HHG. The obtained results for the HH photon number per pulse and wavefront show the potential of using laser systems with $\mathrm{Yb}$ amplifiers for simultaneously creating plasmas and a probe based on HHG. Additionally, the relatively long pulse duration is advantageous for the plasma creation for X-ray lasers, which can be seeded by HHG to achieve a higher brilliance [15].

Acknowledgments: Work funded by Portuguese Fundação para a Ciência e a Tecnologia (FCT) under grants SFRH/BH/52419/2013 (PDF APPLAuSE), SFRH/BD/68865/2010, IF/01122/2013 - CP1163/CT0006, COST Action MP1203, LaserLab European Community's Seventh Framework Programme under grant agreement No. 284464, and Euroatom research and training program 20142018 under grant agreement 633053. This work was supported by the European Union's Horizon 2020 research and innovation programme (VOXEL H2020-FETOPEN-2014-2015-RIA 665207).

Author Contributions: J.C.P.K., S.K., T.W., J.D., G.O.W., P.Z. and M.F. developed the idea and design of the experiment. C.P.J, H.P., V.H, M.G., N.G, J.M.D., N.L, and G.F. prepared the laser for the experiment. B.K. and E.P. provided the calibrated wavefront sensor for the experiment. J.C.P.K., S.K., T.W., and B.K. performed the experiment and data acquisition. J.C.P.K., S.K., T.W. and B.K. analysed the data. J.C.P.K. and S.K. wrote the first draft of the manuscript. All authors participated in preparatory discussions and the manuscript improvement.

Conflicts of Interest: The authors declare no conflict of interest. The founding sponsors had no role in the design of the study; in the collection, analyses, or interpretation of data; in the writing of the manuscript, and in the decision to publish the results.

\section{References}

1. Lee, R.W.; Baldis, H.A.; Cauble, R.C.; Landen, O.L.; Wark, J.S.; Ng, A.; Rose, S.J.; Lewis, C.; Riley, D.; Gauthier, J.C.; et al. Plasma-based studies with intense X-ray and particle beam sources. Laser Part. Beams 2002, $20,527-536$.

2. Zastrau, U. Innovative XUV and X-ray Plasma Spectroscopy to Explore Warm Dense Matter. Ph.D. Thesis, Friedrich-Schiller-Universität Jena, Jena, Germany, 2002.

3. Lindl, J.D.; Amendt, P.; Berger, R.L.; Glendinning, S.G.; Glenzer, S.H.; Haan, S.W.; Kauffman, R.L.; Landen, O.L.; Suter, L.J. The physics basis for ignition using indirect-drive targets on the National Ignition Facility. Phys. Plasmas 2004, 11, 339-491.

4. Saumon, D.; Chabrier, G.; Wagner, D.J.; Xie, X. Modelling pressure-ionization of hydrogen in the context of astrophysics. High Pressure Res. 2000, 16, 331-343.

5. Kluge, T.; Gutt, C.; Huang, L.G.; Metzkes, J.; Schramm, U.; Bussmann, M.; Cowan, T.E. Using X-ray free-electron lasers for probing of complex interaction dynamics of ultra-intense lasers with solid matter. Phys. Plasmas 2014, 21, 033110.

6. Attwood, D.T. The Soft X-Ray And Extreme Ultraviolet Regions of the Electromagnetic Spectrum. In Soft $X$-rays and Extreme Ultraviolet Radiation: Principles and Applications; Cambridge University Press: New York, NY, USA, 2007.

7. Dobosz, S.; Doumy, G.; Stabile, H.; D’Oliveira, P.; Monot, P.; Réau, F.; Hüller, S.; Martin, P. Probing Hot and Dense Laser-Induced Plasmas with Ultrafast XUV Pulses. Phys. Rev. Lett. 2005, 95, 025001.

8. Hergott, J.F. XUV interferometry using high-order harmonics: Application to plasma diagnostics. In Atoms, Solids, and Plasmas in Super-Intense Laser Fields; Springer: New York, NY, USA, 2001.

9. Emma, P.; Akre, R.; Arthur, J.; Bionta, R.; Bostedt, C.; Bozek, J.; Brachmann, A.; Bucksbaum, P.; Coffee, R.; Decker, F.-J.; et al. First lasing and operation of an ångstrom-wavelength free-electron laser. Nat. Photonics 2010, 4, 641-647. 
10. Young, L.; Kanter, E.P.; Krässig, B.; Li, Y.; March, A.M.; Pratt, S.T.; Santra, R.; Southworth, S.H.; Rohringer, N.; DiMauro, L.F.; et al. Femtosecond electronic response of atoms to ultra-in tense X-rays. Nature 2010, 466, 56-61.

11. Zhao, K.; Zhang, Q.; Chini, M.; Wu, Y.; Wang, X.; Chang, Z. Tailoring a 67 attosecond pulse through advantageous phase-mismatch. Opt. Lett. 2012, 37, 3891-3893.

12. Ravasio, A.; Gauthier, D.; Maia, F.R.N.C.; Billon, M.; Caumes, J.-P.; Garzella, D.; Géléoc, M.; Gobert, O.; Hergott, J.-F.; Pena, A.-M.; et al. Single shot diffractive imaging with a table top femtosecond soft X-ray laser harmonics source. Phys. Rev. Lett. 2009, 103, 028104.

13. Williams, G.O.; Chung, H.-K.; Vinko, S.M.; Künzel, S.; Sardinha, A.B.; Zeitoun, Ph.; Fajardo, M. Method of time resolved refractive index measurements of X-ray laser heated solids. Phys. Plasmas 2013, $20,042701$.

14. Morlens, A.-S.; Gautier, J.; Rey, G.; Zeitoun, Ph.; Caumes, J.-P.; Kos-Rosset, M.; Merdji, M.; Kazamias, S.; Cassou, K.; Fajardo, M. Submicrometer digital in-line holographic microscopy at $32 \mathrm{~nm}$ with high-order harmonics. Opt. Lett. 2006, 31, 3095-3097.

15. Zeitoun, P.; Faivre, G.; Sebban, S.; Mocek, T.; Hallou, A.; Fajardo, M.; Aubert, D.; Balcou, P.; Burgy, F.; Douillet, D.; et al. A high-intensity highly coherent soft X-ray femtosecond laser seeded by a high harmonic beam. Nature 2004, 431, 426-429.

16. Constant, E.; Garzella, D.; Breger, P.; Mével, E.; Dorrer, Ch.; Le Blanc, C.; Salin, F.; Agostini, P. Optimizing High Harmonic Generation in Absorbing Gases: Model and Experiment. Phys. Rev. Lett. 1999, 82, 1668.

17. Vuong, C.V.; Khuong, B.D.; Peter, H.; Dao. V. Lap Influence of absorption in phase-matched high-order harmonic generation in a semi-infinite gas cell. J. Nonlinear Opt. Phys. Mater. 2015, 24, 1550031.

18. Gautier, J.; Zeitoun, P.; Hauri, C.; Morlens, A.-S.; Rey, G.; Valentin, C.; Papalarazou, E.; Goddet, J.-P.; Sebban, S.; Burgy, F.; et al. Optimization of the wave front of high order harmonics. Eur. Phys. J. D 2008, 48, 459-463.

19. Künzel, S.; Williams, G.O.; Boutu, W.; Galtier, E.; Barbrel, B.; Lee, H.J.; Nagler, B.; Zastrau, U.; Dovillaire, G.; Lee, R.W.; et al. Shot-to-shot intensity and wavefront stability of high-harmonic generation. Appl. Opt. 2015, $54,4745-4749$.

20. Morrissey, F.X.; Fan, T.Y.; Miller, D.E.; Rand, D. Picosecond kilohertz-class cryogenically cooled multistage Yb-doped chirped pulse amplifier. Opt. Lett. 2017, 42, 707-710.

21. Baumgarten, C.; Pedicone, M; Bravo, H.; Wang, H.; Yin, L; Menoni, C.S.; Rocca, J.J.; Reagan, B.A. 1 J, 0.5 kHz repetition rate picosecond laser. Opt. Lett. 2016, 41, 3339-3342.

22. Kazamias, S.; Douillet, D.; Weihe, F.; Valentin, C.; Rousse, A.; Sebban, S.; Grillon, G.; Augé, F.; Hulin, D.; Balcou, P. Global optimization of high harmonic generation. Phys. Rev. Lett. 2003, 90, 193901.

23. João, C.P.; Pires, H.; Figueira, G. Hybrid diode-pumped Yb:CaF2/YAG laser system delivers $20 \mathrm{~mJ}$ pulses at $1050 \mathrm{~nm}$ for Nd:glass amplifier seeding. OSA Tech. Dig. 2014, doi:10.1364/ASSL.2014.AM5A.45.

24. Flöter, B.; Juranić, P.; Kapitzki, S.; Keitel, B.; Mann, K.; Plönjes, E.; Schäfer, B.; Tiedtke, K. EUV Hartmann sensor for wavefront measurements at the Free-electron Laser in Hamburg. New J. Phys. 2010, 12, 083015.

25. Keitel, B.; Plönjes, E.; Kreis, S.; Kuhlmann, M.; Tiedtke, K.; Mey, T.; Schäfer, B.; Mann, K. Hartmann wavefront sensors and their application at FLASH. J. Synchrotron Radiat. 2016, 23, 43-49.

26. Ammoso, M.V.; Delone, N.B.; Krainov, V.P. Tunnel ionization of complex atoms and of atomic ions in an alternating electromagnetic field. Sov. Phys. JETP 1986, 64, 1191-1194.

27. Henke, B.L.; Gullikson, E.M.; Davis, J.C. X-ray interactions: Photoabsorption, scattering, transmission, and reflection at $\mathrm{E}=50-30,000 \mathrm{eV}, \mathrm{Z}=1-92$. At. Data Nucl. Data Tables 1993, 54, 181-342.

28. Gartside, L.M.R.; Tallents, G.J.; Rossall, A.K.; Wagenaars, E.; Whittaker, D.S.; Kozlová, M.; Nejdl, J.; Sawicka, M.; Polan, J.; Kalal, M.; et al. Extreme ultraviolet interferometry of warm dense matter in laser plasmas. Opt. Lett. 2010, 35, 3820-3822.

29. Ping, Y.; Correa, A.A.; Ogitsu, T.; Draeger, E.; Schwegler, E.; Ao, T.; Widmann, K.; Price, D.F.; Lee, E.; Tam, H.; et al. Warm dense matter created by isochoric laser heating. High Energy Density Phys. 2009, 6, 246-257.

30. Kettle, B.; Dzelzainis, T.; White, S.; Li, L.; Dromey, B.; Zepf, M.; Lewis, C.L.S.; Williams, G.; Künzel, S.; Fajardo, M.; et al. Experimental measurements of the collisional absorption of XUV radiation in warm dense aluminium. Phys. Rev. E 2016, 94, 023203. 
31. Hornung, M.; Liebetrau, H.; Keppler, S.; Kessler, A.; Hellwing, M.; Schorcht, F.; Becker, A.; Reuter, M.; Polz, J.; Körner, J.; et al. $54 \mathrm{~J}$ pulses with $18 \mathrm{~nm}$ bandwidth from a diode pumped chirped-pulse amplification laser system. Opt. Lett. 2016, 41, 5413-5416.

32. Siebold, M.; Roeser, F.; Loeser, M.; Albach, D.; Schramm, U. PEnELOPE: A high peak-power diode-pumped laser system for laser-plasma experiments. Proc. SPIE 2013, 8780, doi:10.1117/12.2017522. 